\title{
A PROPÓSITO DEL ESTUDIO Y EDICIÓN DE MARC ZUILI SOBRE EL FACSÍMIL DEL DICCIONARIO BILINGÜE ESPAÑOL-FRANCÉS Y FRANCÉS-ESPAÑOL DE CÉSAR OUDIN
}

\author{
Francisco Gimeno MenéndeZ \\ Universidad de Alicante \\ fgimeno@ua.es
}

Recibido: 08/08/2017

Aceptado: 08/11/2017

En sus notas para la historia de la lexicografía española, J. Fernández Sevilla (1974: 44) aludió a que la lexicografía hispánica estaba por hacer, y señalaba la dificultad de obtener datos que permitieran formarse una idea justa acerca del estado actual de la lexicografía de su tiempo. Es más, escribió que las manifestaciones más antiguas de la lexicografía parecían estar encaminadas a la recopilación y explicación de términos que (debido al cambio lingüístico y cultural) habían dejado de utilizarse por los diferentes grupos sociales, y por consecuencia se habían hecho extraños e incomprensibles. A este propósito, obedecieron los glosarios de la antigüedad y de la Edad Media, después llamados diccionarios (primero bilingües y después monolingües). La lexicografía románica nació de la necesidad de explicar el significado de los términos, aunque las glosas fueron revisiones y adecuaciones (y hay glosas latinas y glosas romances), más que traducciones y comentarios, y estaban inscritas dentro del contexto social, cultural y temporal de una planificación implícita de los romances, como la mayoría de las normalizaciones lingüísticas.

En los comienzos de la Edad Moderna, la cultura renacentista y la introducción de la imprenta dieron un enorme impuso a la lexicografía. G. Haensch (1982: 106-107) en la tipología de las obras lexicográficas expuso que el primer tipo de obra lexicográfica fue el diccionario bilingüe, y muy pronto también el plurilingüe. En 1490, A. Fernández de Palencia publicó el primer diccionario de latín con explicaciones en castellano, bajo el título de Universal vocabulario. Dos años más tarde, vio la luz el Vocabulario de romance en latín de E. A. de Nebrija, primer diccionario de la lengua castellana que superó al de Fernández de Palencia, en el que se planteó de un modo trascendente la planificación explícita del castellano, con la codificación léxica del estándar castellano, que (junto a la codificación sintáctica de E. A. de Nebrija) materializó el español estándar. Frente al diccionario general monolingüe de la tradición lexicográfica se encontraba el tipo especial del diccionario

Para citar estas notas / To cite this notes: Gimeno Menéndez, Francisco (2017). A propósito del estudio y edición de Marc Zuili sobre el facsímil del diccionario bilingüe español-francés y francésespañol de César Oudin. ELUA, 31: 351-360. doi: 10.14198/ELUA2017.31.18

Enlace / Link: http://dx.doi.org/10.14198/ELUA2017.31.18 
descriptivo de uso restringido, cuya finalidad fue ayudar a quienes estudiaban una lengua extranjera para utilizar correctamente un vocabulario no demasiado extenso.

Posteriormente, M. Alvar Ezquerra (1995: 16-26) en los diccionarios del español en su historia ha aludido a publicaciones recientes y a nuevos proyectos de gran envergadura que cambiarán nuestros conocimientos de manera inmediata. Bien entrado en el siglo XV, el primer vocabulario conocido de nuestra lengua apareció bajo el título de Vocablos dificiles del castellano (manuscrito $\mathrm{n}^{\mathrm{o}} 73$ (12-7-2) de la colección Salazar y Castro en la Real Academia de la Historia), y recogió en una copia muy posterior un centenar y medio de términos que trataban de fijar el uso recto y desterrar incorrecciones. Mientras que las raíces medievales del Universal vocabulario de Fernández de Palencia entroncaron con el saber enciclopédico medieval y la tradición isidoriana, el Lexicón latino-español de E. A. de Nebrija rompió con la tradición medieval latina, y fue el primero en darnos un diccionario moderno, así como marcó la pauta de una renovación en lexicografía que habían de seguir los autores de repertorios bibliográficos posteriores.

La lexicografía de los inicios del siglo XVI en Europa se vio marcada por la publicación de diccionarios plurilingües, reimpresos una y otra vez. El español apareció en los diccionarios plurilingües europeos, no solo por el interés de la lengua o la importancia de sus antecedentes lexicográficos, sino también porque en Bruselas se formó una corte con hispanohablantes, y había un verdadero interés por aprender nuestra lengua, junto al francés y al flamenco, así como al omnipresente latín renacentista. La enseñanza de lenguas distintas del latín durante los siglos XVI y XVII respondía a necesidades comerciales, políticas, etc., y estimuló la aparición de determinados repertorios plurilingües. Así pues, aparecieron en los Países Bajos diccionarios plurilingües y bilingües, como el de H. Hornkens y algunas de las ediciones de los diccionarios de español-francés de J. Palet o de C. Oudin. La ciudad de Amberes adquirió una notable importancia durante varios siglos, tanto como centro de enseñanza de lenguas, como de impresión de los primeros manuales. El Tesoro de las dos lenguas española y francesa de César Oudin fue considerado durante mucho tiempo como la cumbre de la lexicografía hispanofrancesa del siglo XVII por su originalidad.

1. El estudio y la edición de Marc Zuili es mucho más que una introducción críticolexicográfica de un diccionario descriptivo de uso restringido, puesto que ha ofrecido una amplia visión de la preocupación etnográfica y de lingüística aplicada al contacto de lenguas de C. Oudin, sobre la situación sociocultural de la España renacentista. La elaboración de gramáticas, diálogos bilingües, volumen de proverbios, textos bilingües y traducciones literarias estaba dedicada a la ampliación de la competencia comunicativa de los diferentes grupos sociales de la comunidad de habla que aprendían una lengua extranjera.

La publicación de César Oudin, Tesoro de las dos lenguas española y francesa. Tresor des deux langues françoise et espagnole (Editions Honoré Champion, París, 2016) consta de dos volúmenes. El primero comienza con un prólogo, y aparece después dividido en dos apartados. La primera parte consta de una introducción del profesor Marc Zuili y de tres capítulos, seguidos de una conclusión, tres apéndices (documental, índice onomástico e índice de obras citadas), listado de abreviaciones y de referencias bibliográficas. La segunda parte está dedicada a la edición de la totalidad del Tesoro de las dos lenguas española y francesa. Tresor des deux langues françoise et espagnole (a partir de ahora Tesoro) de César Oudin: la parte español-francés figura en el volumen I, y la parte francés-español figura en el volumen II.

El prólogo es de la profesora D. Reyre de la Universidad de Toulouse-Jean Jaurès, quien expone brillantemente el auténtico valor de los diccionarios bilingües, en la medida en que no es tanto la visión de un mundo lo que se propone, sino la transición de una cultura a 
otra. En efecto, la presente edición está muy documentada, y precedida de un largo estudio, no solo sobre esta obra y su autor, sino también sobre la abundante producción de este. Las palabras no fueron las que cambian la lengua, sino la sociedad entera quien evolucionaba hacia otros derroteros. La función de los primeros diccionarios bilingües fue la necesidad que tuvieron los diferentes grupos sociales de una comunidad de conectar una cultura a otra y de comunicarse con otros grupos sociales de dicha comunidad. La propuesta de edición del profesor M. Zuili es, pues, de gran interés, y marcará un hito tanto para la investigación lexicográfica, como para la de las relaciones de civilización e historia entre Francia y España.

2. En la introducción, Marc Zuili (a partir de ahora lo citaré como MZ) alude a la necesidad absoluta de recurrir a los instrumentos esenciales que constituyeron los diccionarios antiguos, sobre todo cuando se trataba de aclarar ciertos pasajes a veces oscuros de los textos españoles del siglo de Oro, en este sentido un muy importante auxiliar fue el Tesoro de César Oudin. Con respecto a la última edición de la parte español-francés del Tesoro, había sido realizada por el profesor B. Pottier (1968), y se trataba de un facsímil de la edición lionesa de dicho diccionario (1675). Su "Presentación" de dos páginas y media dejaba insatisfecho al lector, quien no podía disponer de todas las informaciones que habría deseado sobre esta obra y su autor. Además, estaba totalmente agotada, y únicamente su consulta podía hacerse en las bibliotecas que la habían adquirido.

El estudio es un relevante análisis del contexto sociopolítico y cultural, y tiene tres capítulos y una conclusión. En el primero, MZ presenta las facetas desconocidas hasta ahora de la vida y obra de César Oudin (a partir de ahora CO), sin olvidar la considerable aportación de su propio hijo (Antoine), quien después de la muerte de su padre (1625) participó muy activamente en el enriquecimiento y actualización de sus trabajos. Así pues, ofrece la vida de $\mathrm{CO}$, quien hasta ahora no había sido objeto de una verdadera síntesis, y permitía numerosas lagunas personales y profesionales. Además, contiene algunos datos sobre su hijo Antoine Oudin, cuya obra es indisociable de la de su padre. MZ ha accedido a unos documentos inéditos provenientes de archivos nacionales, que le han permitido aumentar sustancialmente los conocimientos sobre el que se presentaba como "secretario intérprete del rey en lengua alemana, italiana y española". Es más, era también indispensable describir un breve marco del contexto sociopolítico de la época, sobre todo en lo que concernía a las relaciones entre las coronas de Francia y España.

En un tiempo en el que una Francia se encontraba desgarrada interiormente y en conflicto con su vecina España, se desarrolló una gran parte de la vida de CO. Incluso su hijo Antoine (quien continuó la obra paterna) conoció a su alrededor las enemistades y las guerras que enfrentaron a estos dos países. A pesar de que las diferencias que existían entonces eran bien conocidas, MZ ha sentido la necesidad de evocar aquí brevemente la época del fin de las guerras de religión. Cuando CO publicó la primera obra dedicada al español, su Grammaire et observations de la langue espagnole recueillies et mises en françois (1597), la predisposición por esta lengua existía ya desde hacía mucho tiempo en Francia. Sin embargo, se sintió obligado a justificar su posición para evitar las sospechas de tener simpatías por el enemigo español.

A comienzos del siglo XVII, España representaba siempre para los franceses un país que disfrutaba de una inmensa gloria literaria, y las grandes obras del patrimonio literario español (gracias a las corrientes humanistas del Renacimiento) habían sido traducidas al francés desde principios del siglo XVI y ampliamente difundidas. Algunas de ellas habían dado lugar a traducciones sucesivas (así, por ejemplo la Tragicomedia de Calisto y Melibea vulgarmente llamada Celestina), o habían conocido múltiples reimpresiones (lo que atestiguaba su éxito), e incluso esta tendencia prosiguió con el mismo vigor a lo largo del siglo XVII. Así, por ejem- 
plo, las múltiples piezas de teatro de autores franceses de la época (desde las más famosas a las menos) se inspiraron directamente en obras españolas, así como en lo que concernía a la prosa.

Además, la guerra entre los dos países acababa con el motivo de tratados, como el de Vervins (1598), a favor de matrimonios interdinásticos, tal como el que en 1615 unió a Luis XIII y a Ana de Austria, hija de Felipe III de España, aunque sus relaciones fueran poco cordiales. Un año más tarde apareció la nueva edición del Tesoro, la segunda de $\mathrm{CO}$, quien veía el mismo día la prueba evidente de un renovado interés de los franceses por las "cosas de España", sin duda suscitado por el reciente matrimonio real y por la paz establecida entre los dos países. Años más tarde, en 1660, el matrimonio de Luis XIV con María Teresa de Austria, hija de Felipe IV de España, motivó un nuevo interés por el estudio de la lengua española, y prolongó durante todo el siglo XVII el atractivo por nuestra lengua, hasta el punto de superar al italiano como lengua extranjera. Es más, el estudio del español se puso de moda, y formaba parte de los signos externos de la buena educación, así como en la Francia del siglo XVII era normal la utilización del español en los grupos socioculturales medio-altos.

3. Este entusiasmo tuvo también como consecuencia directa un importante desarrollo de la enseñanza del español. Este fenómeno quedó atestiguado por la presencia de un número creciente de autores (españoles o franceses, profesores o especialistas de dicha lengua), y por la multiplicación de publicaciones didácticas (obras consagradas a la gramática castellana, diccionarios bilingües, colecciones bilingües de proverbios, guías de conversación, etc.). Entre los profesores de origen español, MZ recoge las publicaciones de A. de Salazar, J. de Luna, J. de Texeda y S. Deza Sotomayor. Entre los especialistas franceses cita las aportaciones de J. Chapelain, G. Ménage, A. Oudin, J. Saulnier, C. Dupuy des Rosiers y J, Pallet, quien publicó un Diccionario muy copioso de la lengua española y francesa. Dictionaire très ample de la langue espagnole et françoise (París, 1604 y Bruselas, 1606), precursor del Tesoro de $\mathrm{CO}$ y del que supo inspirarse muy hábilmente, no solo con la retención de muchos de sus materiales lexicográficos, sino también con la imitación de la presentación tipográfica sobre dos columnas (un texto español frente a su traducción francesa). En este contexto social y cultural extremadamente favorable a las cosas de España, el cual había favorecido también la multiplicación de traducciones francesas de obras castellanas, CO tomó una parte activa en la puesta a punto de materiales pedagógicos destinados a la enseñanza del español, y como prueba estuvo el número importante de las obras que publicó en este ámbito, donde conoció un inmenso éxito con un número considerable de reediciones a lo largo del siglo XVII y después de su muerte.

Además, en este capítulo, MZ recoge que en 1610, tras la publicación de las primeras ediciones de la mayoría de las obras destinadas a la enseñanza del español, CO vino a España, puesto que juzgaba útil la actualización de los conocimientos "sobre el terreno", con el fin de evitar los peligros de una ciencia demasiada libresca. Un curioso documento publicado por él mismo en una edición bilingüe, como Diálogos muy apazibles escritos en lengua española y traduzidos en francés (1622), describió las diferentes etapas de ese largo viaje a través de España y Portugal. Comentaba, entre otras cosas, que la gente de Navarra era en general favorable al francés, mucho más que la gente de Cataluña. Había bellas librerías en Medina del Campo, y después de visitar Segovia, en la que admiraba la casa de la moneda, el acueducto romano y los tejidos (especialidad artesanal de la villa), franqueó la sierra de Guadarrama para llegar a Madrid.

La corte de la capital de España decepcionó totalmente a $\mathrm{CO}$, quien observó los excesivos gastos de la burocracia oficial, y estaría a favor de que el rey de Francia gastara más 
para entretener a sus pajes y criados de a pie. Sin duda alguna, no debía haberse percatado de la ingente necesidad de subordinados para hacer frente a la colonización y administración de Hispanoamérica, a la que Castilla llevó su experiencia repobladora de la época medieval. Camino de Portugal pasó por Alcalá de Henares, Toledo, Mérida y Badajoz, donde constató que bien pocos viajeros circulaban por este itinerario, y encontraba mejor el viaje entre Orléans y Paris que toda su estancia en España. Atravesó Andalucía, y no dijo nada sobre Sevilla, pero se maravilló delante de la mezquita de Córdoba. Camino de Francia, atravesó el reino de Valencia, llegó a Zaragoza, y visitó Montserrat, Barcelona, Gerona, Perpignan y Salses (frontera), en la que criticó una vez más el sistema aduanero español. En el curso de este viaje que duró seis semanas, $\mathrm{CO}$ compró diferentes libros en castellano, de los cuales más tarde iba a sacar un gran partido, con la edición en Francia o su traducción. Se trataba principalmente de Don Quijote, La historia de los dos leales amantes Theágenes y Chariclea (novela de Heliodoro de Emesa, traducida al español en el siglo XVI por el toledano Fernando de Mena), y de la Galatea de Cervantes. Además, debía añadirse su buen conocimiento de la literatura española (El Lazarillo de Tormes, La Celestina, El examen de los ingenios de Huarte, las obras de Guevara, la obra cervantina, etc.).

4. El segundo capítulo de este estudio está consagrado precisamente a las otras publicaciones de $\mathrm{CO}$, aparte del Tesoro. En efecto, este diccionario se inscribía en un proyecto pedagógico de la enseñanza del español, y no constituía más que una parte del conjunto didáctico más vasto elaborado por este autor. La preocupación esencial de MZ no solo ha consistido en proveer un catálogo tan exhaustivo como fuera posible de las otras publicaciones de $\mathrm{CO}$, sino en establecer la relevancia detallada de las ediciones que han conocido, lo que nunca había sido hecho hasta el presente. Entre estos materiales, MZ ha tenido en cuenta:

a) una gramática española, destinada a procurar las estructuras de la lengua. Así, p. ej., dentro de ese interés por el contenido y la historia de sus ediciones, recoge una lista cronológica de 29 diferentes ediciones atestiguadas (aparecidas en vida de autor y todas ellas del siglo XVII), con un resumen descriptivo y (cuando ha sido posible) con unas indicaciones precisas sobre la localización de estas obras en las diversas bibliotecas francesas y extranjeras;

b) una colección de diálogos bilingües (español-francés), que permitían la adquisición de vocabulario y modismos;

c) un volumen que suministraba unos proverbios españoles, necesarios para el enriquecimiento de la expresión en las situaciones de las conversaciones coloquiales (ya se sabía cuántos eran, y todavía los españoles son muy aficionados a ellos);

d) unos textos bilingües, previstos para una iniciación progresiva a la lectura de obras en castellano, así, p. ej., La conversion d'Athis et de Cloride - La conversión de Atis $y$ de Clorilda (texto original en francés con traducción española vis a vis);

e) unas obras literarias españolas para aquellos que tenían un nivel bastante avanzado, destinadas a unos lectores franceses deseosos de enfrentarse directamente a los textos en lengua española, así como para superar las dificultades de encontrarlas en el comercio francés, así, p. ej., La silva curiosa de Julián Medrano o La Galatea y El curioso impertinente de Miguel de Cervantes, y

f) textos franceses traducidos en español, destinados a un público francés y para el placer de los eruditos españoles, así, p. ej., tradujo un texto francés en verso de Claude Garnier que trasladó en prosa española, le Portrait du très-chrestien roy de France Louys XIII. 
Además, este conjunto se completó con la primera traducción al francés de la primera parte del Quijote de M. de Cervantes, la cual publicó CO en 1614, con inmediato éxito, y seguida de siete nuevas reimpresiones a lo largo del siglo XVII. A pesar de que parece que CO albergó la idea de una traducción de la segunda parte de la obra maestra de Cervantes, dicho tomo fue traducido al francés por el polígrafo François de Rosset en 1618. No es hasta 1625 que las dos traducciones juntas vieron la luz por primera vez (Paris, J. Mestais), y se trataba de la primera edición francesa completa de Don Quichotte. Después, en 1639 la obra completa de Cervantes se presentó al público francés en la casa del editor A. Coulon, con la traducción de CO en un primer tomo y la de F. de Rosset en un segundo. En el siglo XVII las dos traducciones fueron publicadas conjunta e indisociablemente. Aunque la traducción de CO fue criticada por su extrema literalidad y por las importaciones y sustituciones españolas (así como por sus galicismos en el Tesoro), su propuesta tuvo el mérito (entre otras cosas) de poner a disposición de los lectores franceses de comienzos del XVII un gran texto al que muchos no habrían podido acceder, por no saber leer el castellano, así como efectivamente familiarizó a los franceses con el ingenioso hidalgo. Además, publicó una gramática italiana, que tuvo pronto éxito y numerosas reediciones, incluso por mucho tiempo después de la muerte del autor, completada por su hijo Antoine.

Sin duda alguna, la preocupación de MZ en este capítulo por la descripción y comprensión de la extensa obra del brillante lexicógrafo es exhaustiva y enciclopédica, sin descuidar la necesaria complementariedad explicativa de algunas aportaciones, como las principales características del conjunto de ocho Diálogos bilingües de CO: antigua tradición clásica, polémica sobre su autoría, número de ediciones, evolución sin grandes cambios, contenido temático, gran riqueza léxica y paremiológica, consideraciones sobre el estilo y la dimensión cultural, y evaluación de la cualidad de las traducciones en francés propuestas por $\mathrm{CO}$ y después por su hijo A. Oudin.

Aparte de la riqueza de su contenido léxico que reflejaba innegablemente la realidad sociocultural y lingüística de su tiempo (con la aparición de términos como hideputa, vellaco, puto...), los diálogos intentaron reflejar una realidad conversacional, lo cual daba al conjunto un aspecto natural y un estilo sencillo en el que la ironía, el humor y el sarcasmo estaban presentes, dentro de una sintaxis y vocabulario bastante comunes, aunque no impedía que el discurso de los personajes estuviera salpicado muy a menudo de referencias cultas de las literatura española. Más allá de simples textos pragmáticos y didácticos, estos diálogos respondían a unas necesidades comunicativas, que ofrecían a sus lectores esquemas socioculturales de conducta sistematizados bajo el modelo de culturema. Por supuesto que dichos diálogos influyeron en la elaboración de la versión aumentada del diccionario de CO. Sin embargo, deberíamos evitar cualquier ambigüedad entre unos diálogos didácticos (muy próximos al estándar) con el registro coloquial, ya que se trata de diferentes registros (escrito frente a oral) que se refieren no solo a la selección del canal de comunicación, sino también a elecciones vinculadas con la diversidad de los procesos sociales.

5. El tercer capítulo se dedica exclusivamente al Tesoro, sin olvidar que fue un texto pionero de la lexicografía hispano-francesa y de gran amplitud. Aquí MZ expone minuciosa y sistemáticamente la historia completa de las ediciones de esta obra, publicada por vez primera en 1607 por CO, y ampliada después de unos años, lo que le permitió valiosas soluciones y esquemas. Después de su muerte (1625) conoció correcciones y adiciones bien al cuidado de su hijo Antoine (quinta edición de 1645 del Tesoro), bien a la del editor Jean Mommart ( $2^{\mathrm{a}}$ edición bruselense realizada en 1660). En la edición parisina de 1660 y en la edición lionesa de 1675 de este mismo diccionario, unas nuevas entradas fueron introducidas todavía por los 
libreros-impresores que examinaban estos volúmenes sobre el mercado. En conjunto, ocho ediciones sucesivas de esta obra fueron expuestas al público, entre 1607 y 1675, lo cual fue una prueba admirable de su inmenso éxito, que conoció aun tres ediciones suplementarias realizadas después de la muerte de su hijo. $\mathrm{MZ}$ se ha esforzado en ofrecer un listado de todas las versiones del Tesoro, y proveer una descripción detallada de cada una de estas publicaciones, completada por la trascripción integral de las partes preliminares que allí figuran.

Entre las resoluciones, MZ cita la importancia del contenido de cada "Advertencia necesaria a los lectores", que figuraba al comienzo de los diferentes volúmenes consultados. En función del año de la publicación, dicho contenido era modificado por $\mathrm{CO}$, quien seguía muy de cerca las evoluciones del sistema fonológico del español entre la segunda mitad del siglo XVI y la primera mitad del XVII, las cuales fueron testigo del paso del sistema fonológico medieval al moderno. En particular, $\mathrm{CO}$ dejó constancia del reajuste fonológico, con la información a sus lectores de las variaciones grafemáticas del ensordecimiento y confusión de las sibilantes, y por consiguiente la comparación de las diferentes advertencias ofrecía a los historiadores de la lengua unos datos muy valiosos. Además, el estudio minucioso de cada una de las ediciones del Tesoro le ha permitido a MZ un excelente listado sistemático de las variantes aparecidas en el curso de las ediciones, y el análisis de estas evoluciones le ha llevado al establecimiento de un stemma de las diferentes ediciones de la obra, el cual permite plantear de un modo esquemático toda la historia del Tesoro.

Asimismo, MZ se ha planteado unos análisis sobre las fuentes, donde $\mathrm{CO}$ extrajo sus materiales, y la amplia influencia que ejerció sobre numerosos lexicógrafos que utilizaron abundantemente sus propios trabajos, y a veces los plagiaron. Entre los autores anteriores y mejores lexicógrafos de su tiempo, cita los trabajos lexicográficos de E. A. de Nebrija, Cristóbal de las Casas, H. Hornkens y J. Pallet, y constituían una buena prueba de la exigencia y preocupación de $\mathrm{CO}$ por recoger los mejores trabajos lexicográficos de su tiempo, dentro del ámbito de la lengua española. Desde la segunda edición de su diccionario en 1616, CO no vaciló en proponer en anexo el conjunto del Vocabulario de germanía, publicado por primera vez en España en el año 1609, y de integrar unos mil términos salidos directamente del diccionario monolingüe (Tesoro de la lengua castellana o española) de Sebastián de Covarrubias, cuya edición príncipe fue de 1611.

En fin, MZ también se ha interesado por los numerosos lexicógrafos que a su alrededor más o menos largamente encontraron su inspiración en el Tesoro, y explica cuál es la deuda contraída con CO, a saber: Girolamo Vittori, Lorenzo Franciosini, César-Joachim Trognesius, Arnaldo De la Porte, Nicolas Mez de Braidenbach y Esteban de Terreros. En el siglo XVIII, F. Sobrino (antiguo oficial del ejército de Flandes), como ya había hecho con la Grammaire espagnole y con el conjunto de los diálogos de CO, se apropió sin escrúpulos y sin mencionarlo de la casi totalidad del Tesoro, y la publicó bajo su nombre en Bruselas con el título de Diccionario nuevo de las dos lenguas española y francesa (1705).

En particular, L. Franciosini fue el autor de numerosas obras, y coincidió con $\mathrm{CO}$ en la publicación de una gramática, un vocabulario, unos diálogos y una traducción del Quijote a su lengua. Entre ellas, sobresalió uno de los más célebres diccionarios bilingües italiano-español y español-italiano de los siglos XVII y XVIII. La edición en dos volúmenes se publicó en Roma (1620), y muchos de sus materiales fueron extraídos directamente de la edición parisina de 1616 del Tesoro de CO. A pesar de que la crítica especializada había tildado la obra de L. Franciosini de meras traslaciones al italiano de lo que había realizado anteriormente $\mathrm{CO}$ en francés, J. J. Martínez Egido (2003) realizó un estudio pormenorizado de ambas obras, con 
la finalidad de establecer el grado de dependencia del autor italiano con respecto al francés. La comparación entre los dos diccionarios se realizó sobre una selección de dos muestras diferentes de estudio, según se atendía a las macroestructuras o a las microestructuras de las obras. La conclusión constató que la construcción de artículos se presentaba de forma diferente en las dos obras., y que no era acertado colegir que la obra del lexicógrafo italiano dependía de la obra del lexicógrafo francés. L. Franciosini debía conocer y consultar la obra de CO de 1615 , pero más como un referente para perfilar los materiales, que como una fuente directa de la que extrajera una información básica. En efecto, M. Alvar Ezquerra (2002: 218) aludió a que la extensión del Vocabolario (así como la manera de elaborarlo) y el empleo que hacía L. Franciosini de las obras de sus predecesores dejaron bien claro que no tuvo una fuente única, los cuales venían a demostrar una importante actividad y profesionalidad lexicográfica.

Además, MZ ha añadido unas páginas en este capítulo sobre una actuación de $\mathrm{CO}$, desde 1609 , en una línea de doble plagio de ediciones trilingües, cuya característica principal era la introducción del italiano al lado del francés y del español por parte de G. Vittori (Tesoro de las tres lenguas francesa, italiana y española), a quien había reprobado claramente, pero que finalmente supo utilizarlo hábilmente a su favor, con el enriquecimiento de más de 3000 entradas que no figuraban en su volumen anterior. Y MZ cierra este capítulo con un excelente cuadro de esquema general (pág. 162), en el que sintetizaba los lazos de unión entre la obra lexicográfica de $\mathrm{CO}$ y los numerosos diccionarios citados en este apartado, tanto anteriores como posteriores al Tesoro, con el fin de visualizar la filiación (sin duda compleja) de estas obras, con la caracterización de la importancia experimentada y ejercida por cada uno de ellos.

6. Finalmente, en la conclusión de este estudio MZ repasa sintéticamente los puntos más importantes, y también le permite explicar por qué ha hecho la elección de proponer el facsímil de su edición bruselense de 1660, dentro del marco de la presente publicación del Tesoro. Hasta ahora era difícil para los lectores de hoy acceder a una versión del Tesoro que presentara de manera rigurosa la exigencia de unos criterios científicos. Las ediciones anteriores no se podían consultar más que en algunas bibliotecas, y la versión facsimilar de B. Pottier (1968) estaba agotada desde hacía mucho tiempo. En cuanto a las versiones electrónicas en Internet carecían de la debida presentación científica. Por consiguiente, era muy importante una nueva edición con un estudio original, comprensivo y explicativo, basado sobre las investigaciones recientes que reconstruyeran la historia lingüística, social y cultural de esta obra y de su autor, lexicógrafo, gramático, paremiólogo, profesor y traductor. En la nueva edición se ha seleccionado la edición facsimilar de Bruselas (publicada por J. Mommart en 1660) por la gran cualidad de su impresión de origen, y por el cuidado extremo que ha presidido en su realización.

Asimismo, MZ ha descubierto ciertas facetas de la existencia de CO, como autor de las numerosas obras consagradas (la mayoría a la enseñanza del español), y continuada por su hijo Antonio, hasta su muerte. No obstante, entre todas sus publicaciones, el Tesoro ocupó un puesto preferente (no solo por su amplitud), que le consagró como el primer gran diccionario bilingüe para el uso del español y del francés en el siglo XVII, así como le otorgó un capítulo en la historia de los diccionarios de la lengua. Desde el siglo XVI, el interés por el castellano había dado ya lugar a la realización de múltiples traducciones de obras literarias españolas, y esta tendencia no hizo más que acentuarse en el curso del siglo siguiente.

A dicha conclusión sigue un amplio apéndice documental que da al lector la posibilidad de acceder al conjunto de las obras preliminares de las diferentes ediciones del Tesoro, reunidas aquí por primera vez. Contiene igualmente la trascripción de las páginas 
intituladas "Ampliaciones y correcciones para el cuerpo del libro", que curiosamente no aparecían más que en ciertos ejemplares de la edición del Tesoro, realizada en París en 1645 por A. Oudin. Gracias a esta trascripción los lectores de la presente edición dispondrán de materiales lexicográficos suplementarios que completarán las de la edición facsímil que ha propuesto.

A dicho apéndice, sigue un índice onomástico, un índice de obras citadas y una exhaustiva bibliografía (documentos de archivos, diccionarios, obras de búsquedas bibliográficas, inventario de bibliotecas, obras generales sobre la lexicografía española, obras antiguas, y obras y artículos modernos de erudición), que permitirán conocer mejor no solamente la vida y obra de CO, sino también la historia de sus publicaciones, y en particular la de su obra maestra, el Tesoro, joya de la lexicografía bilingüe franco-española de la época y accesible de nuevo en nuestros días en su integridad.

7. Dentro de la lexicografía hispano-francesa, según D. Azorín (2001: 70-9), el francés y el español poseían una larga tradición lexicográfica que se remontaba a la primera mitad del siglo XVI. La primera obra que podemos considerar como auténtico diccionario fue el Recueil de dictionaires Françoys, Espagnolz et Latins de H. Hornkens, publicado en Bruselas en 1599, aunque no era un diccionario bilingüe estricto, sino un diccionario trilingüe monodireccional, cuya lengua de entrada fue el francés, seguida de la traducción española y de su equivalente en latín. Sus materiales fueron ampliamente aprovechados por dos de los autores de mayor renombre en el ámbito de la lexicografía plurilingüe del siglo XVII: J. Palet y sobre todo C. Oudin.

Con el Tesoro de las dos lenguas española y francesa. Tresor des deux langues françoise et espagnole (1607), CO culminó su trayectoria la lexicografía hispano francesa, aunque hubo posteriormente otros lexicógrafos. La importancia de la labor descriptiva que llevó a cabo CO sobre el español de los Siglos de Oro no solo se debió a la calidad de sus obras y a sus excelentes dotes de lexicógrafo y gramático, sino también al momento histórico del tránsito entre los siglos XVI y XVII, en que estas aparecieron. El talento de CO como lexicógrafo hizo que superara a cuantos le habían precedido en el terreno de la lexicografía bilingüe, de quienes supo aprovechar lo mejor de sus aportaciones.

Sobre los problemas de la definición o equivalentes por traducción en el diccionario bilingüe, R. Werner (1982: 285-7) comentó que si fuera así podría dar la impresión de que los diccionarios bilingües sirvieran exclusivamente (o en gran parte) como instrumento para la traducción. En teoría podría establecerse una gran diferencia entre la explicación lexicográfica del diccionario semasiológico monolingüe y la del diccionario de traducción bilingüe, la cual consistía en que aquel describía qué contenidos podían corresponder a determinados significantes de una lengua, y en este qué significantes de una lengua de destino podían corresponder a determinados significantes de una lengua de origen. En efecto, los diccionarios bilingües se usaban con frecuencia no solo como instrumentos para la traducción, sino del mismo modo que los diccionarios semasiológicos y onomasiológicos.

Para el usuario del diccionario bilingüe no se trataba (en muchos casos) de traducir, con ayuda de este enunciados posibles o realizados a otra lengua, sino de comprenderlos o producirlos, a partir de los conceptos, ya que el problema teórico fundamental de la traducción consistía en que las estructuras léxicas de distintas lenguas no se correspondían, y nunca era posible encontrar en la lengua de destino un conjunto de significantes al que correspondía un contenido que se compusiera, desde un punto de vista semasiológico, exactamente de los mismos elementos que el contenido correspondiente al conjunto de 
significantes dado en la lengua de origen. En este sentido, el ideal del traductor (que explica el exhaustivo estudio de MZ) no podía consistir en producir en la lengua de destino un texto, cuyo contenido correspondiera exactamente al contenido del texto de la lengua de origen, sino más bien un texto cuyo contenido concordaba con el mayor número posible de elementos esenciales con el contenido del texto de la lengua de origen.

Además, en el diccionario descriptivo debíamos superar las críticas sobre las transferencias lingüísticas y algunos presupuestos obsoletos sobre dos sistemas lingüísticos en cierto modo cerrados, a tenor de ciertos presupuestos metodológicos (neogramáticos y estructuralistas), ya que las lenguas no fueron resultados, sino actuaciones comunicativas. No se trataba de una descripción léxica de dos estructuras abstractas, ya que respondían a instrumentos comunicativos esenciales del desarrollo cognitivo del ser humano, en los que el contexto bilingüe favorecía las transferencias lingüísticas, sociales y culturales, y en particular las variables lingüísticas de la importación y la sustitución (cambio de código y calco) entre las lenguas implicadas. Las situaciones sociales de contacto de lenguas favorecen la comunicación de los diferentes grupos sociales, dentro de las diversas comunidades de habla, y en general la convergencia lingüística, social y cultural, así como forman parte de las relaciones entre lengua, cultura y sociedad. La integración lingüística y la social de las importaciones y sustituciones constituyeron el gran capítulo de los préstamos en la lengua francesa y española. Sin duda alguna, el excelente estudio y edición del profesor MZ ha confirmado que el Tesoro de CO fue el mayor proyecto hasta entonces en el terreno de la lexicografía bilingüe española, y el modelo para los numerosos lexicógrafos de los siglos XVII y XVIII.

En general, podría afirmarse que en el mundo hispánico se disponía de menos diccionarios y otras obras lexicográficas, en comparación con otras lenguas importantes, tales como el inglés, el francés, el alemán y el ruso. A causa de la importancia creciente de la lexicografía en el mundo actual, la bibliografía sobre esta materia ha aumentado considerablemente en los últimos años, y es absolutamente necesario que en el ámbito hispánico se incrementen tanto los resultados de nuevas ediciones sobre obras lexicográficas plurilingües, como las investigaciones de la lexicografía aplicada. Por supuesto que la contribución de este estudio y edición del profesor MZ es extraordinaria, y sintetiza una labor y preocupación de varios años. Su propuesta de edición es muy brillante, y marcará la investigación lexicográfica del futuro.

\section{Referencias bibliográficas}

Alvar Ezquerrra, M. (1995), "Los diccionarios del español en su historia", en De antiguos y nuevos diccionarios del español, Madrid, Arco/Libros, 2002, págs. 5-50.

Alvar Ezquerrra, M. (2002), "El Vocabolario italiano e spagnolo de Lorenzo Franciosini”, en De antiguos y nuevos diccionarios del español, Madrid, Arco/Libros, págs. 191-220.

Azorín, D. (2004), Los diccionarios del español en su perspectiva histórica, Alicante, Universidad de Alicante.

Fernández Sevilla, J. (1974), Problemas de lexicografia actual, Bogotá, Instituto Caro y Cuervo.

Haensch, G. (1982), “Tipología de las obras lexicográficas”, en Haensch, G. et al. (1982), págs. 95-187. Haensch, G. et al. (1982), La lexicografia. De la lingüistica teórica a la lexicografia práctica, Madrid, Gredos.

Martínez Egido, J. J. (2003), "El Tesoro de Oudin (1607/1616), y el Vocabulario de Franciosini (1620): dos diccionarios bilingües del español", en Con A. Zamora Vicente (Actas del Congreso Internacional "La lengua, la Academia, lo popular, los contemporáneos...”, Alicante, Universidad de Alicante, págs. 815-823.

Werner, R. (1982), "La definición lexicográfica”, en Haensch, G. et al. (1982), págs. 259-328. 